\title{
Modelling of Thermal and Gas-Dynamic Processes in the Porous Structures
}

\author{
M.N. Gaynullin, V.A. Sotova, P.A. Chertykovtsev , E.O. Barbonov, S.V. Zaika \\ Samara University, Heat engineering and heat engines Department, Samara, Russia
}

\begin{abstract}
In this article is considered the use of porous material in heat exchangers. The programming model with a porous regenerator heat exchanger was compiled in the program ANSYS Fluent complex. The paper does comparative analysis of influence of different material porosity on flow parameters.
\end{abstract}

\section{Introduction}

In recent years, there is an important issue about creating a highly efficient compact heat exchangers for different purposes in many branches of industry including aviation, space, missile and laser.[1,2] At the same time, emerging tasks can be successfully solved only in case of the intensification of heat transfer processes [3].

One of the most promising and effective ways of heat intensification exchange processes is the use of heat exchangers porous metals. The physical basis of this method is in the high intensity of heat transfer between the metal frame and flowing coolant through it due to the high surface area of contact and effective mixing in the pores $[4,5,6]$.

A wide range of porous materials properties, the ease of fabrication of these structural elements, the high intensity of heat - all these aspects enable to use the porous materials in diversified conditions.

Currently using of various technologies gives reasons for the creation of porous materials with different structures. For the above-mentioned areas the highly heatcarrier structures are in the great interest of technology.

Thermal and hydraulic calculation of heat exchangers on the basis of porous structures is carried out using mass conservation equations, pulse strength and energy with appropriate initial and boundary conditions. The mathematical model in general formulation is rather complicated and has no exact analytical solutions. That is the reason various kinds of assumptions and empirical relations for transfer coefficients in the equations and boundary conditions are introduced.

One of the most important parameters is the porosity of the material which is determined by the ratio of void volume to total volume of the porous body. The ideal environment is called a continuous porous plate which is pierced in the direction of the filtering system of cylindrical channel of the same diameter; its length is equal to the plate thickness. Real porous structure has significant features. The pores usually constitute a curved channel variable cross-section, both in form and in size.

For grabbing information about the hydraulic resistance mechanism and intensity of heat transfer at the moment of moving cooler in porous structures for the closure of a mathematical model, extensive experimental studies are needed. And if the characteristics of the structures of small and medium porosity and heat and mass transfer processes in these studied enough, these questions are not studied deeply enough for the high porosity structures.

\section{The dynamic processes in porous structures calculation methodology}

At the present time the CAE-systems are used for modeling of thermal and gasdynamic processes and for calculation of machines made of porous materials. [7]

Table 1. Calculated model initial parameters

\begin{tabular}{|l|l|l|l|l|}
\hline Element & \multicolumn{1}{|c|}{$\begin{array}{c}\text { Ra- } \\
\text { dius, } \mathbf{~ m}\end{array}$} & $\begin{array}{c}\text { Length, } \\
\mathbf{~ m}\end{array}$ & $\begin{array}{c}\text { Wall } \\
\text { thickness, } \mathbf{~}\end{array}$ & Material \\
\hline $\begin{array}{l}\text { The cold } \\
\text { heat } \\
\text { exchanger }\end{array}$ & $1 \cdot 10^{-2}$ & $2,5 \cdot 10^{-2}$ & $1,07 \cdot 10^{-3}$ & Copper \\
\hline $\begin{array}{l}\text { Regene- } \\
\text { rator }\end{array}$ & $8 \cdot 10^{-3}$ & $14 \cdot 10^{-2}$ & $1,07 \cdot 10^{-3}$ & $\begin{array}{l}\text { The } \\
\text { glass } \\
\text { tube }\end{array}$ \\
\hline $\begin{array}{l}\text { The hot } \\
\text { heat } \\
\text { exchanger }\end{array}$ & $\begin{array}{l}2,4 \cdot \\
10^{-2}\end{array}$ & $2 \cdot 10^{-2}$ & $1,65 \cdot 10^{-3}$ & Copper \\
\hline \begin{tabular}{l} 
Receiver \\
\hline
\end{tabular} & $2,6 \cdot 10^{-2}$ & $1,49 \cdot 10^{-1}$ & $5 \cdot 10^{-3}$ & $\begin{array}{l}\text { Stainless } \\
\text { steel }\end{array}$ \\
\hline
\end{tabular}




\subsection{The programme modelling in the program ANSYS Fluent complex}

The programming model with a porous regenerator heat exchanger in the program ANSYS Fluent complex was compiled on the basis of a study of dependencies operating parameters of the design and other work. The model is a two-dimensional model of a longitudinal section, built in the "Compass 3D» (Figure 1). The model geometric parameters are presented in Table 1. It is needed to be mentioned that the system is closed. Pressure within the system is $0.1 \mathrm{MPa}$. Air in the system under the influence of the alternator Q-drive enters the cold heat exchanger, whereby the temperature drops.

For model work it is necessary to set the input parameters values of the calculated installation, such as the average pressure, frequency, volume rate, etc. (Table 2 ). The crank radius is set to $4 \mathrm{~mm}$ and the piston stroke $8 \mathrm{~mm}$.

Table 2. The model initial parameters at the entrance.

\begin{tabular}{|c|c|c|}
\hline Parameters & $\begin{array}{c}\text { Designation } \\
\text { ANSYS Fluent }\end{array}$ & Value \\
\hline $\begin{array}{c}\text { Frequency, } \mathrm{Hz} \\
\begin{array}{c}\text { The initial gas } \\
\text { temperature, } \mathrm{K}\end{array}\end{array}$ & $\mathrm{Freq}$ & 50 \\
\hline $\begin{array}{c}\text { The initial pressure } \\
\text { value, } \mathrm{Pa}\end{array}$ & $\mathrm{Pa}$ & $0.101325 \cdot 10^{6}$ \\
\hline
\end{tabular}

Then air is drawn into a glass tube, wherein the porous insert is mounted. After insertion of the porous air enters the hot heat exchanger, where the temperature rises. Then, the air enters the receiver. The pressure in the receiver increases, while the pressure in the cavity to cool heat exchanger is relatively small.

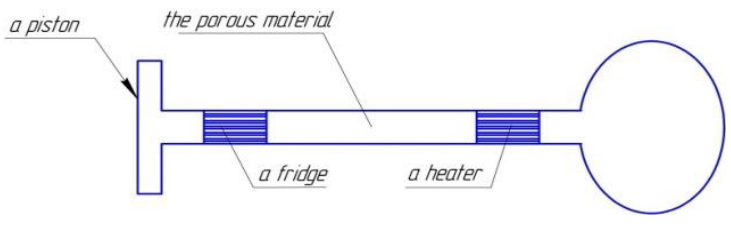

Figure 1. Estimated model regenerator.

Ansys Fluent software was used to modeling the processes in the cooler. There was built two-dimensional model, was made the appropriate settings of the boundary conditions. The model with a dynamic mesh was used to simulate the compressor piston vibrations. To set the piston motion in Ansys Fluent was used option «InCylinder», allowing to set the required piston motion law. Using this option the calculation step has been set according to the crank angle and was 1 degree. Using PC with a processor Intel Core I7-4770K $3.5 \mathrm{GHz}, 32 \mathrm{~GB}$, SSD 240GB calculation time of each calculation model was about 60 hours. On figure 1 and figure 2 shows the fields of total pressure in top dead centre and in bottom dead centre tor stainless steel material with porosity 0,6 .

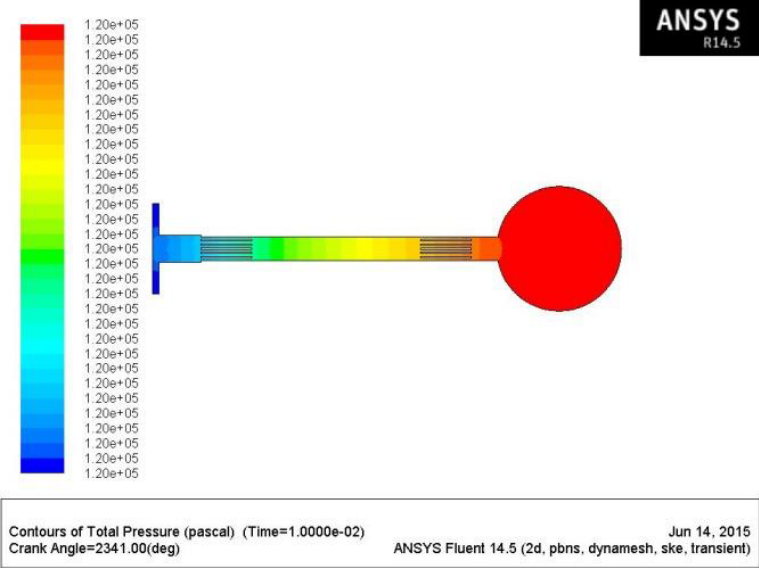

Figure 2. The field of total pressure in top dead centre (material: stainless steel, Porosity: 0,6)

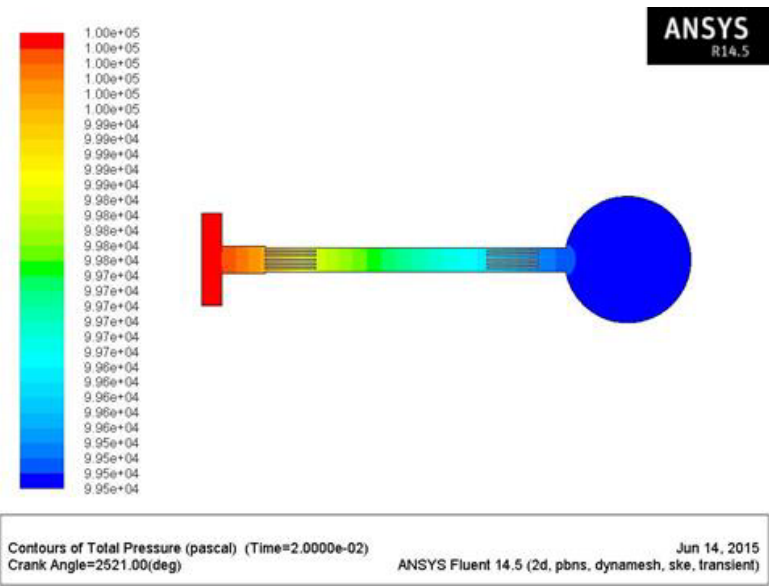

Figure 3. The field of total pressure in bottom dead centre (material: stainless steel, Porosity: 0,6)

\subsection{Calculations and air flow parameters' dependence of the porosity}

The following formula was used for thermal conductivity in a porous medium ratio calculation:

$$
\lambda=\varepsilon \lambda_{f}+(1-\varepsilon) \lambda_{s}
$$

According to the results of calculation, there are the graphs of pressure dependencies of the Reynolds number and velocity of the porosity of the material (Figures 4-6).

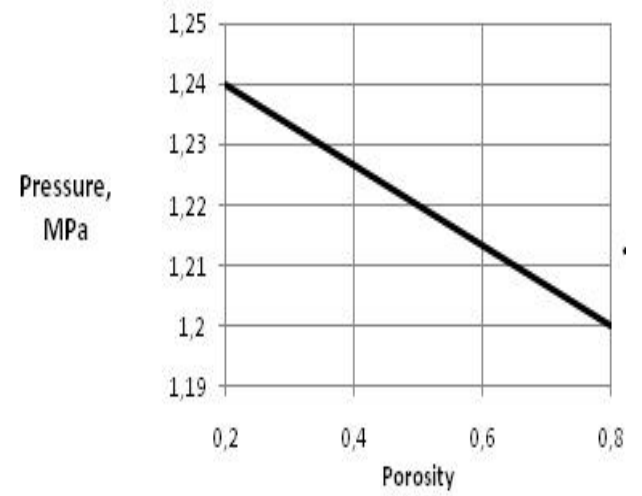

Figure 4. Pressure dependence of the porosity of the material 


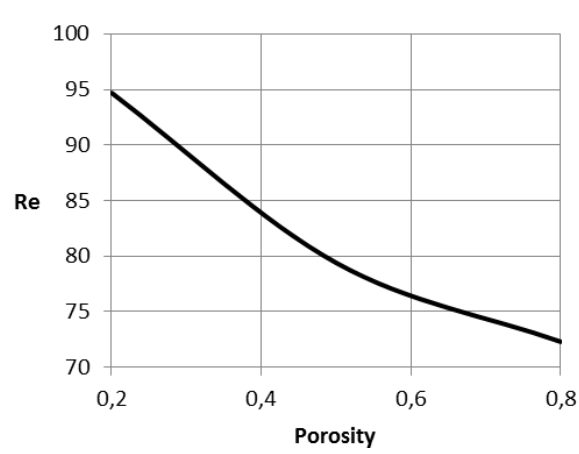

Figure 5. Dependence of Reynolds number on the porosity of the material.

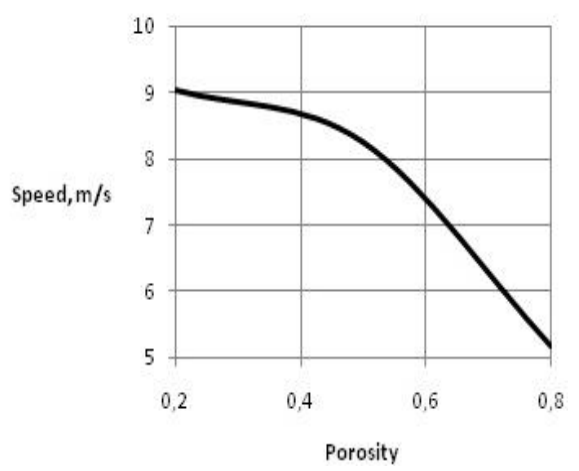

Figure 6. Depending on the porosity of the material speed.

For the most detailed research of the porosity effectiveness of the gas-dynamic processes, additional calculations were carried out using such materials as bronze, brass, stainless steel. During the calculation the coefficient of local loss, the coefficient of friction losses, the differential pressure on the porosity of the materials were obtained (figures 7-11).

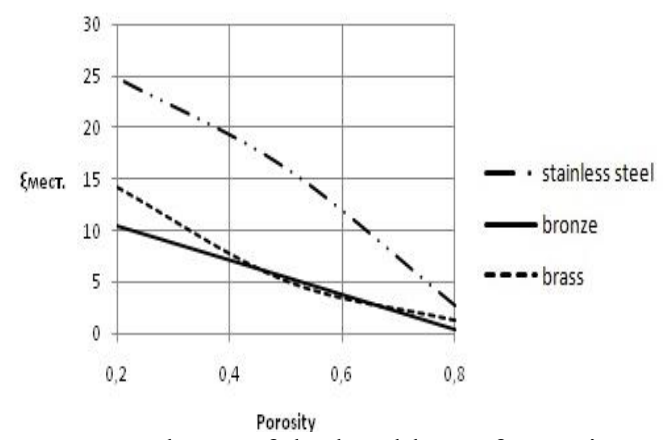

Figure 7. Dependence of the local loss of porosity materials.

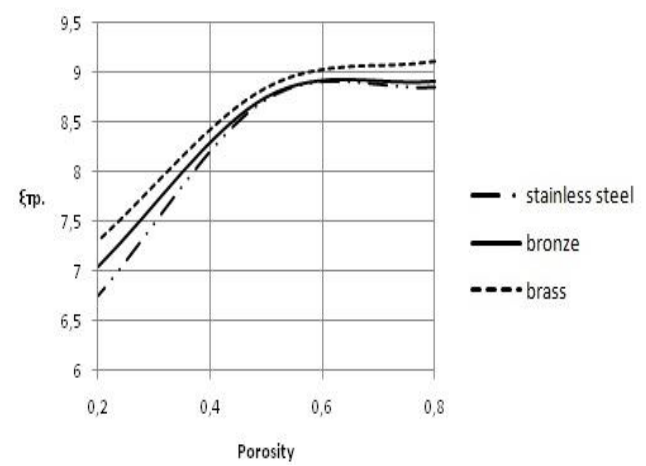

Figure 8. Dependence of loss factor on the porosity of the friction material.

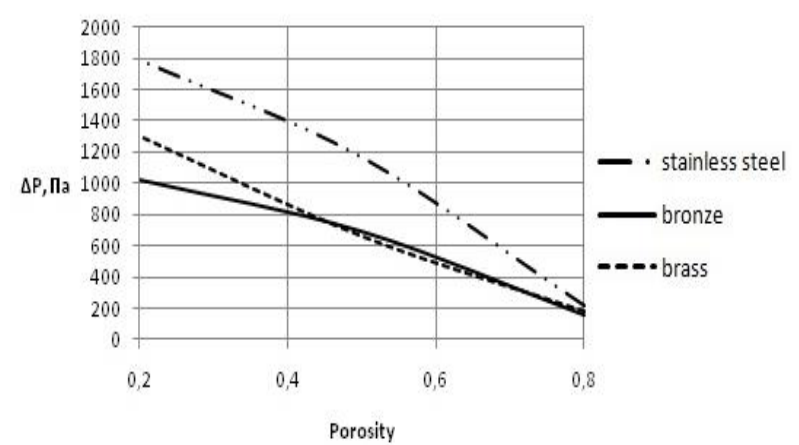

Figure 9. Differential Pressure dependence of the porosity of the material.

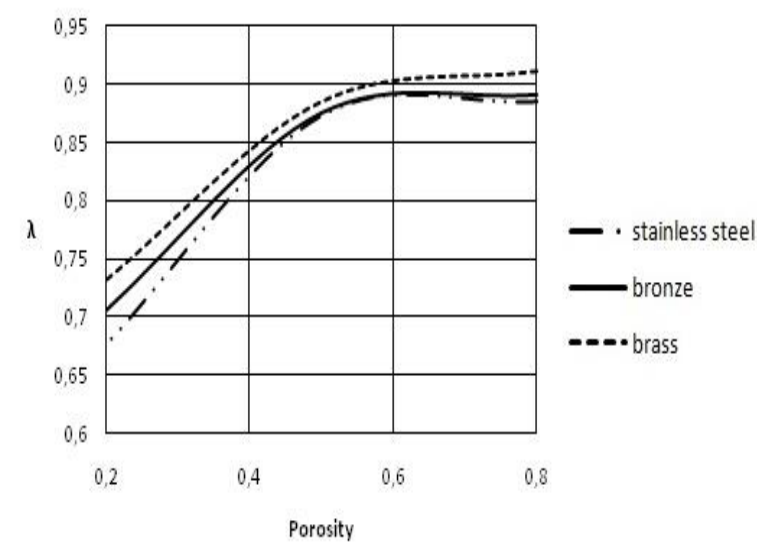

Figure 10. Dependence of the coefficient of hydraulic friction losses on the porosity of materials.

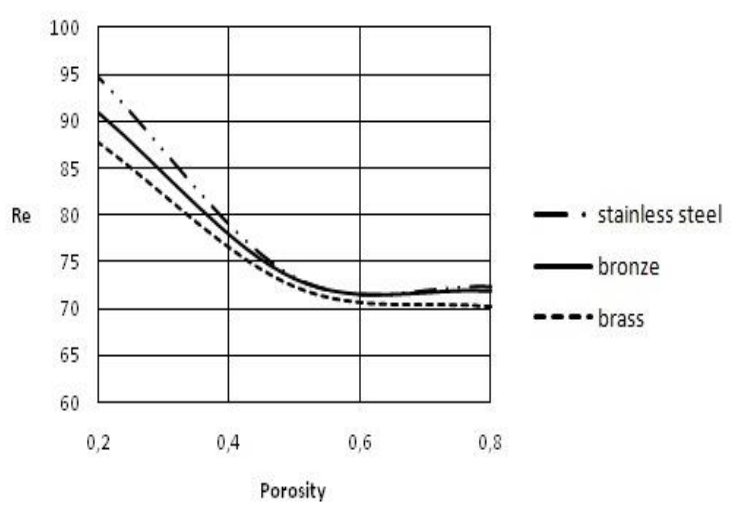

Figure 11. The dependence of the Reynolds number on the porosity of materials

\section{Conclusions}

The following conclusions are based on the results of the calculations:

1. With increased porosity materials differential pressure drops 7-8 times.

2. The loss factor decreases by $97 \%$, taking into consideration the increasing porosity materials, whereas the coefficient of friction loss increases by $25 \%$ with increasing porosity from 0.2 to 0.5 , and changes slightly with increasing porosity from 0.5 to 0.8 .

3 . The hydraulic loss factor increases with porosity from 0.2 to 0.5 by $30 \%$, while the Reynolds number falls quarter. Further changes are minor. 


\section{References}

1. T. Trollier, Air Liquid space cryocooler system, Cryogenics 48, Pp. 267-273, (2008).

2. T. Trollier, T. Ravex, I. Charles, L. Duband, J. Mullié, P. Bruins, T. Benschop, M. Linder, Miniature 50 to $80 \mathrm{~K}$ Pulse Tube Cooler for Space Applications, International Cryocooler Conference, (2002).

3. A. M. Arkharov, Cryogenic systems: basic theory and calculation, Engineering, 464, (1988).

4. I. A. Popov, Gidrodinamika and heat transfer in porous heat exchange elements and devices, 240, (2007).
5. C. S. Kirkconnell, R. C. Hon, T. Roberts Raytheon Stirling/Pulse Tube Cryocooler Maturation Programs Cryocoolers 15, 31-37, (2009).

6. W. Scheirer, T. Roberts, E. Pettyjohn, Multistage Pulse Tube Refrigeration Characterization of the Lockheed-Martin RAMOS cryocooler, Cryocoolers 15, 39-47, (2009).

7. T. R. Ashwin, G. S. V. L. Narasimham and Subhash Jacob CFD analysis of high frequency miniature pulse tube refrigerators for space applications with thermal non-equilibrium model Applied Thermal Engineering, 30, 152-166, (2010). 